\title{
Involvement of dietitians in diabetes care, results from a secondary care hospital in Greece
}

\author{
Zoe Pafili ${ }^{1}$, Sophia Samara ${ }^{2}$, Charilaos Dimosthenopoulos $^{3}$ and Olga Gkortzi ${ }^{2}$ \\ ${ }^{1}$ Department of Dietetics, Evaggelismos General Hospital, Athens, Greece, \\ ${ }^{2}$ Department of Nutrition and Dietetics, Technological Educational Institute of Thessaly, Karditsa, Greece and \\ ${ }^{3}$ Departmenr of Dietetics, Laiko General Hospital, Athens, Greece
}

\section{Abstract}

Introduction: According to diabetes care standards nutrition therapy should be an integral part of diabetes management, and all individuals with diabetes should be referred to a registered dietitian for nutrition therapy at - or soon after-diagnosis and for ongoing follow-up. There is limited international data that indicate that a large percentage of people with diabetes have not received structured diabetes education and have not visited a dietitian. The aim of this study was to assess the involvement of dietitians in diabetes care in Greece.

Materials and Methods: All adult diabetic patients admitted to a secondary care general hospital in Greece during 30 consecutive days were included in the study. Patients admitted in the ICU, CICU, day clinics and hemodialysis patients were excluded. Data were obtained by personal interviews using a 40 item questionnaire which included 10 questions regarding number of visits to dietitians for diabetes management, whether patients were referred by their doctors or sought dietary advice by their own, reasons for visiting a dietitian, goal achievement and patient satisfaction.

Results: In total 124 patients (68 males and 56 females) with diabetes were admitted to the hospital during the study period (4 type 1 , 114 type 2 and 6 pregnancy diabetes). Data were obtained from $3\left(22.8 \pm 6 \mathrm{yrs}, 26.1 \pm 5.7 \mathrm{~kg} / \mathrm{m}^{2}, 8.3 \pm 5.9 \mathrm{yrs}\right.$ with diabetes $), 105(76.6$ $\pm 11.3 \mathrm{yrs}, 28.0 \pm 5.3 \mathrm{~kg} / \mathrm{m}^{2}, 12.8 \pm 9.3 \mathrm{yrs}$ with diabetes), and $5\left(32.6 \pm 4.4 \mathrm{yrs}, 28.5 \pm 4.0 \mathrm{~kg} / \mathrm{m}^{2}\right)$ patients with type 1 , type 2 and pregnancy diabetes respectively. Two out of 3 type 1 diabetes and 1 out of 5 patients with pregnancy diabetes interviewed reported to have been referred to a dietitian by their doctor. Only $5.7 \%$ ( 6 patients) of type 2 diabetes patients reported to have been referred to a dietitian by their doctor and another $5.7 \%$ have visited a dietitian on their own initiative. Five out of 6 referrals were at diabetes diagnosis. The number of encounters with a dietitian ranged from 1 to 24 with patients seeking to loose weight having the greater number of encounters. Of type 2 diabetes patients $94.3 \%$ did not receive lifestyle advice before commencing diabetes medication whereas $25 \%$ did not receive any dietary advice by any health professional even after starting medication.

Conclusions: In our cohort the majority of diabetes patients had not received dietary counseling by a dietitian, whereas about one fourth of type 2 diabetes patients had not received any dietary advice.

\section{Conflict of Interest}

There is no conflict of interest. 